\title{
Spitz melanoma secondary to xeroderma pigmentosa in a child: a case report
}

\author{
Zhengjun Gao, MD, Shilin Chang, $\mathrm{PhD}^{\star}$
}

\begin{abstract}
A 6-year-old boy presented with a 5-year history of pigmentation on the face, neck, and hands. On examination, the patient had extreme photophobia. There was multiple freckles-like pigmentations on the face, neck, and back of both hands. Extremities were densely distributed. Multiple facial lesions, $1-2 \mathrm{~cm}^{2}$ in size, were also seen; most of them were ulcerated and had crusty patches. There was mild double eversion of the lower eyelids, with pigmentation and conjunctival redness in both eyes. Facial histopathologic findings: Spitz nevus-like malignant melanoma, Clark grade IV. A final diagnosis of melanoma secondary to xeroderma pigmentosa was made. According to the previous literature, the minimum age of patients with xeroderma pigmentosum combined with malignant melanoma is 8 years old, but the age, in this case, is 6 years old. In addition, extensive local excision of suspicious lesions early is of great significance for the prognosis of patients.
\end{abstract}

Keywords: Xeroderma pigmentosum, Spitz malignant melanoma, Children, Case report

Xeroderma pigmentosa $(\mathrm{XP})$ is an autosomal recessive genetic disease, and it was the first human disease to be discovered related to DNA damage repair defects ${ }^{[1]}$. About $70 \%$ of the patients have a history of parental consanguinity. In this disease, the DNA damage of the patient's skin cannot be repaired after exposure to ultraviolet rays, and the pyrimidine dimers, produced by ultraviolet rays, cannot be effectively removed. DNA repair genes mutations are often caused, and light damage-related diseases and skin malignancies appear ${ }^{[2]}$. Recently, a 6-year-old boy was admitted to our department. The lesion of face developed into Spitz melanoma from xeroderma pigmentosum.

\section{General information}

The 6-year-old boy was born in Longnan County, Gansu Province. The boy is of Han nationality and Preschool children. No previous treatment. No history of drug allergy and there is no similar medical history in the parents' family. The parents were cousins. The child

Department of Plastic Surgery, Gansu Provincial Hospital, Lanzhou, China

This case has reported work in accordance with SCARE 2018 standards.

This manuscript has been peer reviewed.

Sponsorships or competing interests that may be relevant to content are disclosed at the end of this article.

${ }^{*}$ Corresponding author. Address: Department of Plastic Surgery, Gansu Provincial Hospital, Lanzhou 730000, China. Tel: + 86 15095421737. E-mail address: cs/1035110316@163.com (S. Chang).

Copyright $@ 2020$ The Authors. Published by Wolters Kluwer Health, Inc. on behalf of IJS Publishing Group Ltd. This is an open-access article distributed under the terms of the Creative Commons Attribution-Non Commercial-No Derivatives License 4.0 (CCBY-NC-ND), where it is permissible to download and share the work provided it is properly cited. The work cannot be changed in any way or used commercially without permission from the journal.

International Journal of Surgery Oncology (2020) 5:e88

Received 14 February 2020; Accepted 27 February 2020

Published online 11 May 2020

http://dx.doi.org/10.1097/IJ9.0000000000000088 was taken to the hospital for treatment by his parents. We were told the boy has had photophobia after birth and face and neck stains for 5 years. Freckles-like pigmentation appeared after sun exposure on the face and neck since the age of 1 year, which could merge into dark brown plaques. Facial lesions often rupture, then crust or fall off on their own, forming superficial scars. When ulceration and crusting alternately, hyperkeratosis formed a skin angle. The hypopigmented areas were looked like stockings. The skin was dry on both calves and forearms, with extremely small, pinpoint pigmentations. The area of hypopigmentation in both thighs and upper arms was reduced concentrically. The skin on the trunk was slightly dry, the appearance was normal. The parents had 2 children, both of the children were diagnosed with XP. This case was the older brother (Fig. 1A), the younger brother was 3 years old (Fig. 1B).

\section{Examination}

The child weighed $16.5 \mathrm{~kg}$ and was $100 \mathrm{~cm}$ tall. He was slightly lighter than his peers, slightly weaker in spirit, and normal in intelligence. No superficial lymph nodes swelling throughout the body, and systemic examinations were normal. Dermatological examination showed dry skin, freckles on face, neck, and hands. Facial skin lesions were $1-2 \mathrm{~cm}^{2}$ in size and mostly were ulcerated and crusty. Both eyes showed conjunctival congestion and mild eversion of both lower eyelids. The pigments were distributed in the extremities, below the knees or elbows, they look like mesh stockings (Figs. 1C, D). Laboratory examinations: complete blood count, basic metabolic panel, and full set tumor markers were within the normal limits. Chest $\mathrm{x}$-ray, electrocardiogram, and abdominal ultrasound were also normal. Postoperative pathologic tissue biopsy showed that the surface of the tissue was covered with squamous epithelium, and some areas were eroded. The fullthickness of the dermis showed a diffuse distribution of heterogenous cells rich in pigment and various forms. The epithelial cells and spindle-shaped spindle cells are the dominant cells, with an irregular nucleus, obvious nucleoli, rich chromatin, common 

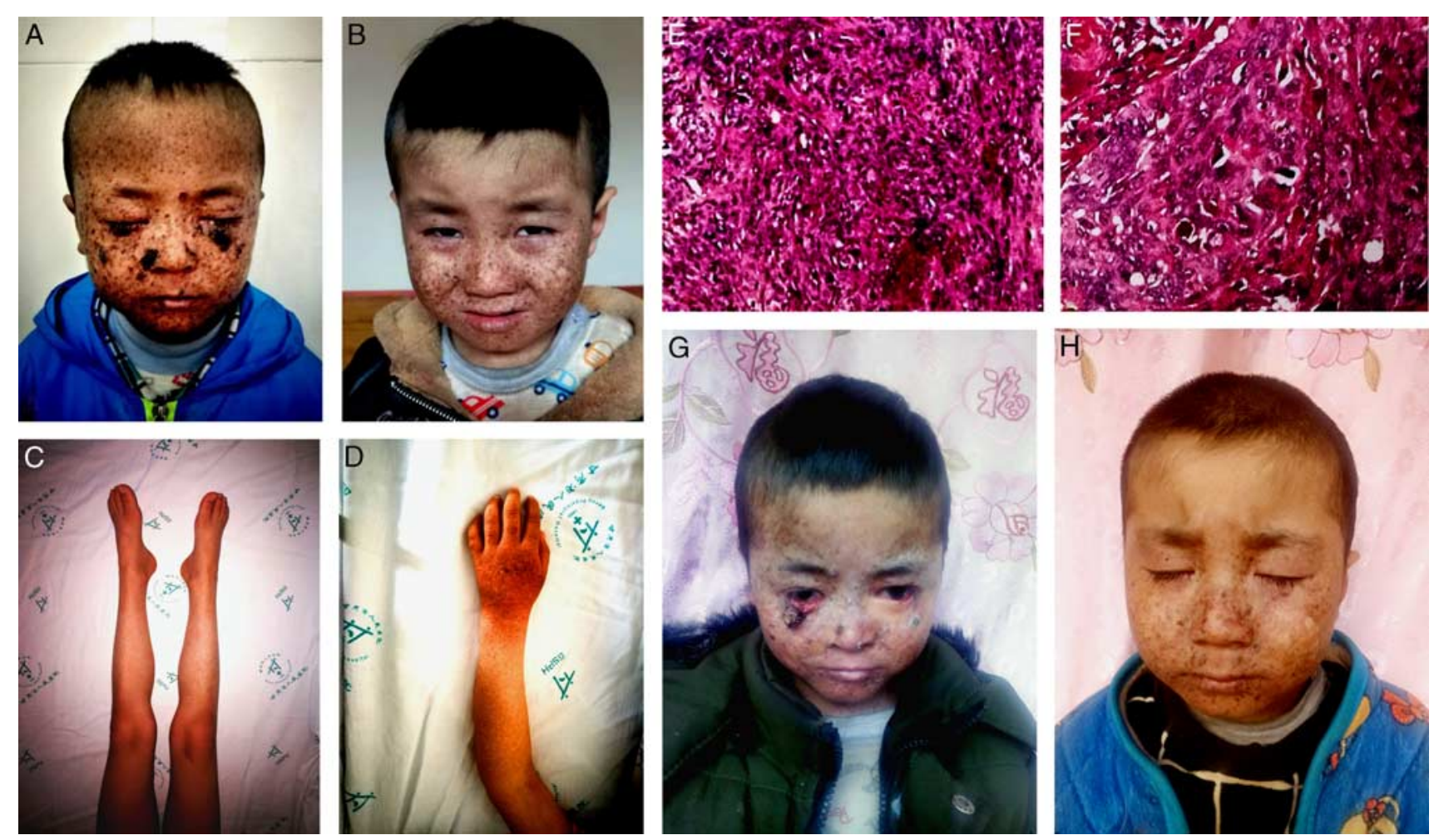

Figure 1. A, Photograph of a sick boy before surgery. B, The boy's younger brother. C, Photograph of his lower limb stains. D, Photograph of his upper limbs. E and F, Microscopic photographs of pathologic tissue biopsies. G, The boy 17 months after surgery. H, The younger brother after 17 months.

division, abundant cytoplasm, and rich in pigment particles. The above histopathologic findings were suggestive of Spitz nevus-like malignant melanoma, Clark grade IV (Figs. 1E, F) and a final diagnosis of melanoma secondary to XP was made.

\section{Methods}

The patient's facial skin melanoma showed repeated signs of ulceration, and related preoperative examinations were completed. No contraindications to surgery or general anesthesia were seen. After confirming that the patient was able to tolerate anesthesia and surgery, extensive resection and suture of the melanoma mass $(0.2 \mathrm{~cm})$ under general anesthesia was given to the boy. Skin grafts were performed with the left groin skin for areas with large surgical defects. Cefazolin sodium pentahydrate $0.5 \mathrm{~g}$ bid intravenously, Agkistrodon acutus hemagglutinase 0.5 g qd into a Moffie tube, tobramycin ointment bid applied to the eyes was given during the perioperative period. After surgery, conventional silicone gel anti-scar treatment was needed, and the whole body skin, eye conjunctiva, and sclera were protected from sunlight. The patient is currently recovering well with no signs of local recurrence. This case is reported in accordance with the SCARE 2018 standard $^{[3]}$, and other detailed reports can be found in the full SCARE list.

\section{Results}

The operation went well and there were no signs of local recurrence, but there were new lesions on the right lower eyelid. Figures $1 \mathrm{G}$ and $\mathrm{H}$ are the status of 17 months post the operation.
There were no adverse complications, all wounds healed in stage 1 , and all skin grafts survived.

\section{Discussion}

The average age of onset is 6 months to 3 years old. Patients mainly have photophobia; conjunctiva is susceptible to irritation and congestion, and severe sunburn occurs, even with a slight exposure to sun light. Freckle-like pigmentation of the skin in the exposed area, poor skin elasticity, dryness and atrophy, may cause hypopigmentation. The exposed area is prone to skin malignancies. The most common skin malignancies are basal cell carcinoma and squamous cell carcinoma, while melanoma is the rarest skin malignancy found to be associated with xeroderma pigmentosum. In XP patients, the average age at the onset of malignancy is 8 years old. However, in our case, melanoma developed at the age of 6 years. About $25 \%$ of patients may present with neurological diseases and mental retardation ${ }^{[4-6]}$. Compared with the common melanoma, the prognosis of Spitz like melanoma is difficult to predict, because of the inconsistency in the diagnosis level of Spitz like melanoma, the reported negative factors affecting the prognosis of Spitz like melanoma, including the elderly, tumor diameter $>1$ $\mathrm{cm}$, mitotic index $>6$ mitotic images $/ \mathrm{mm}^{2}, 9$ p21 allele deletion, TERT promoter mutation, etc ${ }^{[7,8]}$.

In this case, the parents of the patient were cousins, and the clinical manifestations, history and signs, and postoperative pathologic examination supported the diagnosis of XP. The age of onset of skin cancer is only 6 years, and it is the most malignant disease of skin cancer-melanoma, and the prognosis is probably poor. The patient's postoperative pathologic examination was 
suggestive of Spitz nevus-like melanoma, which is a rare pathologic type. Its features include epidermal hyperplasia, epidermal fissures surrounding the dermal cell nests, large epithelioid cells, and bundled spindle cells. Particular attention should be paid to identify the Spitz moles.

Currently, no definitive treatment is available and XP can not be completely cured. The treatment is mostly supportive along with the strict sunlight protection and application of effective light-proofing agents. Early surgical removal of skin lesions is particularly important. Regular examination is crucial to identify the skin malignancies at the earliest and once tumors are found, they should be treated accordingly ${ }^{[9]}$.

\section{Ethical approval}

Written informed consent for publication was obtained from the patient.

\section{Sources of funding}

None declared.

\section{Author contribution}

Z.G. and S.C. contributed the same to this article. They contributed to write the manuscript, to gather the clinical data as well as the relevant imaging information. S.C. did the overall supervision and contributed in finalizing the manuscript. He will also act as a guarantor for the work presented here.

\section{Conflict of interest disclosure}

The authors declare that they have no financial conflict of interest with regard to the content of this report.

\section{Research registration unique identifying number (UIN)}

Not applied.

\section{Guarantor}

Zhengjun Gao.

\section{References}

[1] Cleaver JE. Transcription coupled repair deficiency protects against human mutagenesis and carcinogenesis: personal reflections on the 50th anniversary of the discovery of xeroderma pigmentosum. DNA Repair 2017;10: 21-8.

[2] Sha Y, Vartanian V, Owen N, et al. Modulation of UVB-induced carcinogenesis by activation of alternative DNA repair pathways. Sci Rep 2018; 8:705.

[3] Agha RA, Borrelli MR, Farwana R, et al. For the SCARE Group. The SCARE 2018 Statement: Updating Consensus Surgical CAse REport (SCARE) Guidelines. Int J Surg 2018;60:132-6.

[4] Hui L, Zhifang X, Xiaohong L, et al. One case of infantile pigmented xeroderma with keratoacanthoma. J Clin Exp Pathol 2014;30:941-2.

[5] Kalamkar C, Radke N, Mukherjee A, et al. Xeroderma pigmentosum with bilateral ocular surface squamous neoplasia and review of the literature. BMJ Case Rep 2016;10:111-3.

[6] Sainsbury K, Walburn J, Araujo-Soares V, et al. Challenges and proposed solutions for formative research to inform systematic intervention development in rare and unstudied conditions: the case example of Xeroderma Pigmentosum. Br J Health Psychol 2018;23:229-37.

[7] Yazdan P, Cooper C, Sholl LM, et al. Comparative analysis of atypical spitz tumors with heterozygous versus homozygous 9 p21 deletions for clinical outcomes, histomorphology, BRAF mutation, and p16 expression. Am J Surg Pathol 2014;38:638-45.

[8] Barnhill RL, Flotte TJ, Fleischli M, et al. Cutaneous melanoma and atypical spitz tumors in childhood. Cancer 1995;76:1833-45.

[9] Weon JL, Glass DA. Novel therapeutic approaches to xeroderma pigmentosum. Br J Dermatol 2018;181:249-55. 